\title{
Prolactin-Induced Prostate Tumorigenesis Links Sustained Stat5 Signaling with the Amplification of Basal/Stem Cells and Emergence of Putative Luminal Progenitors
}

\author{
Lucila Sackmann-Sala, ${ }^{*}$ Aurélie Chiche, ${ }^{*}$ Nerea Mosquera-Garrote, ${ }^{*}$ Florence Boutillon, ${ }^{*}$ Corinne Cordier, ${ }^{\dagger}$ Ivan Pourmir, \\ Luz Pascual-Mathey, ${ }^{*}$ Karima Kessal, ${ }^{*}$ Natascha Pigat, ${ }^{*}$ Philippe Camparo, ${ }^{*}$ and Vincent Goffin*
}

Accepted for publication July 10, 2014.

Address correspondence to Vincent Goffin, Ph.D., INSERM U1151, INEM, University Paris Descartes, Faculty of Medicine, 14 rue Maria Helena da Silva - CS 61431, Bâtiment Leriche, Porte 9 (75993) Paris CEDEX 14, France. E-mail: vincent. goffin@inserm.fr.

\begin{abstract}
Current androgen ablation therapies for prostate cancer are initially successful, but the frequent development of castration resistance urges the generation of alternative therapies and represents an important health concern. Prolactin/signal transducer and activator of transcription 5 (STAT5) signaling is emerging as a putative target for alternative treatment for prostate cancer. However, mechanistic data for its role in development or progression of prostate tumors are scarce. In vivo mouse studies found that local prolactin induced the amplification of prostate epithelial basal/stem cells. Because these cells are proposed cells of origin for prostate cancer and disease recurrence, we looked further into this amplification. Our results indicated that sustained Stat5 activation was associated with the occurrence of abnormal basal/stem cell clusters in prostate epithelium of prostate-specific prolactintransgenic mice. Analysis of epithelial areas containing these clusters found high proliferation, Stat5 activation, and expression of stem cell antigen 1. Furthermore, enhanced prolactin signaling also led to amplification of a luminal cell population that was positive for stem cell antigen 1 . These cells may originate from amplified basal/stem cells and might represent important progenitors for tumor development in prostate epithelium. These data provide a deeper understanding of the initial stages of prostate tumorigenesis induced by prolactin to help determine whether this hormone or its downstream messengers could be useful targets for prostate cancer treatment in the future. (Am J Pathol 2014, 184: 3105-3119; http://dx.doi.org/10.1016/j.ajpath.2014.07.020)
\end{abstract}

The role of local prolactin (PRL) in prostate cancer development and/or progression is emerging. ${ }^{1}$ Studies of PRL signaling in prostate tissue or prostate cell lines have found that PRL signals mainly through Janus kinase 2/signal transducer and activator of transcription 5 (Jak2/Stat5) in the prostate. ${ }^{2,3}$ In human prostate cancer biopsies, elevated levels of STAT5 activation were found to correlate with high Gleason scores ${ }^{4}$ and early recurrence. ${ }^{5}$ In addition, PRL expression has been detected in $>50 \%$ of castrate-resistant prostate cancer and metastases. ${ }^{6}$ However, mechanistic data for PRL/Stat5 action in the development or progression of prostate tumors are scarce. We have previously reported that local PRL induces the amplification of the $\mathrm{p} 63^{+}$epithelial basal/stem cell compartment in the mouse dorsal prostate. ${ }^{3}$ Given the proposed role of basal/stem cells as cells of origin for mouse and human prostate cancer ${ }^{7,8}$ and the accumulated evidence from human prostate carcinomas, targeting PRL signaling in the prostate might be an alternative for treatment of this deadly disease. $1,9,10$

As opposed to the human prostate, the mouse prostate is composed of different lobes (ventral, lateral, dorsal,

Supported by Fondation ARC grants PDF20101202327 (L.S.-S.) and SFI20101201635, Fondation de France grant 2011-00020359, La Ligue contre le Cancer grant 13/75-63, Cancéropôle Ile de France and Institut National du Cancer grant INCa_6672.

Disclosures: None declared.

Current address of N.M.-G., Laboratorio de Investigación 8, Instituto de Investigación Sanitaria (IDIS), Hospital Clínico Universitario de Santiago de Compostela (CHUS), SERGAS, Santiago de Compostela, Spain; of L.P.-M., Facultad de Química Farmacéutica Biológica, Universidad Veracruzana, Xalapa, Veracruz, Mexico; of K.K., Equipe S12-Chimiokines et physiopathologie du segment antérieur de l'oeil, Institut de la Vision, UMR S 968 Inserm/UPMC/CNRS 7210, Paris, France. 
anterior). Importantly, individual lobes often vary in terms of signaling and phenotypic characteristics on genetic or experimental manipulation. ${ }^{11}$ Even though the dorsal and lateral lobes (frequently called dorsolateral) are considered more similar to the human prostate, to date it is not clear whether any one particular lobe might be more appropriate to model human disease. ${ }^{1,11}$ In terms of PRL action, some reports suggest a more marked effect of this hormone in dorsal and lateral lobes than in the ventral lobe. . $^{2,13}$

To progress in our understanding of PRL action in the prostate, we have furthered our in vivo studies of the probasin $(P b)-P R L$ mouse model. $P b-P R L$ mice are transgenic for a genetic construct that contains the prostate-specific minimal $\mathrm{Pb}$ promoter to drive the expression of the rat PRL cDNA. ${ }^{14}$ These mice are exposed to increased PRL after late prepubertal stages, when ductal formation and branching is mostly finished. In addition, they display normal circulating testosterone levels. As a result of PRL exposure, $P b$-PRL mice display marked prostate enlargement of ventral, lateral, and dorsal lobes after 10 weeks of age. Both epithelium (distended ducts filled with secretions) and stroma (densely fibrous with increased cellularity) are affected. ${ }^{14}$ We have recently reported that Stat5 activation and cell proliferation are widespread in the epithelium of $P b-P R L$ dorsal prostates, with development of low-grade prostate intraepithelial neoplasia (PIN) lesions at 6 months, evolving to high-grade PIN and occasionally to carcinomas in some animals at old age (approximately 20 months). ${ }^{3}$ In addition, cells in the amplified basal/stem cell layer often appeared to form clusters, where cells were enlarged and sometimes positioned perpendicularly to the basement membrane. ${ }^{3}$

Here, we have analyzed the $P b-P R L$ prostate epithelium at the hyperplastic/low-grade PIN stage in an effort to understand the initial stages of tumor development induced by PRL. The lobe-specific pattern of Stat 5 activation was tightly associated with the prevalence of basal cell clusters in each lobe, more so than with overall cell proliferation. The observed Stat5 activation was exclusively present in luminal cells, strongly suggesting paracrine regulation of the basal/stem compartment by luminal cells. Furthermore, enhanced PRL signaling stimulated the emergence of a new subpopulation of putative luminal progenitor cells that had never been described in wildtype (WT) prostates. These cells might derive from abnormally amplified basal/stem cells and may represent a relevant step in the process of PRL-induced prostate tumorigenesis.

\section{Materials and Methods}

\section{Animals}

$P b-P R L$ mice on the $\mathrm{C} 57 \mathrm{~B} 1 / 6 \mathrm{~J}$ background were generated in the laboratory as previously described. ${ }^{3}$ WT littermates were used as controls. Until sacrifice, mice were kept on a 12/12-hour light/dark cycle with normal food and water provided ad libitum. Prostate samples were obtained by microdissection immediately after sacrifice by cervical dislocation. Unless otherwise stated, all mice were 5 to 9 months of age. Procedures were approved by the local ethical committee for animal experimentation (authorization CEEA34.VG.095.12).

\section{Western Blot Analysis}

Freshly dissected prostate lobes were snap frozen in liquid nitrogen and stored at $-80^{\circ} \mathrm{C}$ until processing. For protein extraction, frozen tissues were homogenized with mortar and pestle, and homogenates were dissolved in lysis buffer supplemented with protease and phosphatase inhibitor cocktail (Thermo Scientific, Waltham, MA). Protein concentration was measured by Bradford assay (Bio-Rad Protein Assay; Bio-Rad, Hercules, CA). Protein samples (20 to $50 \mu \mathrm{g}$ ) were resolved in $4 \%$ to $12 \%$ gradient SDS-PAGE in NuPAGE BisTris Precast Gels (Life Technologies, Carlsbad, CA) or 10\% SDS-PAGE gels. Proteins were then transferred onto nitrocellulose membranes (Bio-Rad) and stained with primary antibodies directed against phospho(p)-Stat5 (9359 or 4322; Cell Signaling Technology, Danvers, MA), total Stat5 (sc835; Santa Cruz Biotechnology, Dallas, TX), p-Stat3 (9131; Cell Signaling Technology), total Stat3 (9132; Cell Signaling Technology), p-extracellular signal-regulated kinase (Erk) 1/2 ( $\mathrm{p}-$ mitogen-activated protein kinase 1/3; 06-182; Millipore, Billerica, MA), total Erk1/2 (9106; Cell Signaling Technology), p-Akt S473 (9271; Cell Signaling Technology), total Akt (9272; Cell Signaling Technology), and mouse PRL polyclonal number 9 (generously provided by Gudmundur Thordarson, Texas Tech University Health Sciences Center, El Paso, TX). For band detection, horseradish peroxidase-coupled secondary anti-rabbit (7074; Cell Signaling Technology) or anti-mouse (NA931; GE Healthcare Europe, Freiburg, Germany) antibodies were added before electrochemiluminescence substrate (Immobilon Western Chemiluminescent HRP Substrate; Millipore; or LumiGLO Reagent; Cell Signaling Technology). For comparisons between WT and $P b-P R L$ prostate lobes, blotting of p-Stat3, total Stat3, p-Stat5, and total Stat5 were performed on the same membranes that were stripped with Restore Plus stripping buffer (Thermo Scientific) and restained. The same was done for p-Akt and total Akt and for p-Erk1/2, total Erk1/2, and PRL.

\section{AlphaScreen SureFire p-Stat5 Assay}

An AlphaScreen SureFire Stat5 (p-Tyr694/699) Assay Kit (PerkinElmer, Waltham, MA) was used to detect p-Stat5 in a semiquantitative manner. This assay is recommended for use in human cellular lysates. To validate its use in mouse prostate tissue lysates extensive testing and optimization procedures were performed through close collaboration with the manufacturer (F. Boutillon et al, unpublished data). The optimized assay involved pretreatment of tissue lysates (obtained as detailed above) with protein-A Sepharose beads to deplete immunoglobulins. Protein concentrations were then 
remeasured, and $2 \mu \mathrm{g}$ of each sample was used. Detection was performed according to the kit instructions, with substitution of AlphaScreen Acceptor Beads with AlphaLISA Acceptor Beads. Samples were tested in duplicate.

\section{Histology and IHC/IF}

Freshly dissected prostates were fixed in $4 \%$ paraformaldehyde and embedded in paraffin. Tissue slides $(4 \mu \mathrm{m})$ were stained with hematoxylin and eosin for histological analysis. The immunohistochemistry/immunofluorescence (IHC/IF) protocol involved deparaffination of tissue slides followed, except for stem cell antigen (Sca)-1 and PRL staining, by heat-induced antigen retrieval in citrate buffer $(\mathrm{pH}$ 6). For IHC, endogenous peroxidases were inactivated with $\mathrm{H}_{2} \mathrm{O}_{2}$. IHC/IF slides were incubated in blocking serum before overnight incubation with primary antibodies or with antibody diluent (MM-France, Francheville, France) for negative controls. Biotinylated antirabbit, anti-rat, or universal (anti-rabbit and anti-mouse) secondary antibodies were used for IHC (Vector Laboratories, Burlingame, CA); avidin-biotin-horseradish peroxidase complex and diaminobenzidine (Vector Laboratories) were added for detection. Slides were counterstained with hematoxylin and analyzed with $10 \times$ and $40 \times$ objectives under an EVOS xl Core digital inverted microscope (Life Technologies). Fluorescently labeled secondary antibodies used for IF were anti-mouse Alexa Fluor 594 (A-11032; Life Technologies) and anti-rabbit Alexa Fluor 488 (A-11034; Life Technologies). Nuclei were stained with Hoechst dye, and slides were analyzed with a $10 \times$ objective under an Axio Observer.Z1 inverted microscope (Carl Zeiss Microscopy, Jena, Germany). Primary antibodies included anti-cytokeratin (CK)-5 (M3270; Spring Bioscience, Pleasanton, CA), CK-8 (MMS-162P; Covance, Princeton, NJ), Sca-1 (557403; BD Biosciences, San Jose, CA), Stat5 (sc-835; Santa Cruz Biotechnology), Ki-67 (RM-9106-S0; Thermo Fisher Scientific, Cheshire, UK), and PRL (A0569; Dako, Glostrup, Denmark).

\section{Basal Cell Cluster Counts and IHC/IF Analysis of Cluster Areas}

Prostate tissue slides stained for CK- 5 by IHC (as detailed in the above paragraph) were analyzed under an EVOS xl Core digital microscope (Life Technologies) for the presence of basal cell clusters. Strict criteria were applied when defining a basal cell cluster as follows: i) four or more $\mathrm{CK}-5^{+}$cells grouped together (in contact or very close); ii) large cells of round rather than flat shape; and iii), different morphology compared with other surrounding CK- $5^{+}$(basal) cells. All cluster counts were performed manually by the same researcher.

Basal cell cluster areas analyzed by IHC/IF included basal cells that formed clusters plus the immediately adjacent luminal cells. First, basal cell clusters were identified on IF slides costained for CK-5 and CK-8. A total of $45 P b-P R L$ clusters ( $n=11$ to 19 clusters from $n=3 P b-P R L$ mice) and $30 \mathrm{WT}$ clusters $(n=4$ to 9 clusters from $n=5 \mathrm{WT}$ mice) were analyzed. Next, epithelial areas, including each identified basal cell cluster and the corresponding neighboring luminal cells, were delineated. The defined areas were analyzed on the CK-5/CK-8 costained slides and on serial slides stained by IHC for Stat5, Sca-1, and Ki-67. According to the staining intensity and the percentage of positive cells in the delimited area, a score from 0 to 5 was attributed to each of Sca-1, Ki-67, and nuclear Stat5 stainings. Scores 0 to 2 and 3 to 5 were later grouped into low and high scores, respectively. CK-5/CK-8 costained slides were also examined for the presence/absence (yes/no) of double positive $\mathrm{CK}-5^{+} \mathrm{CK}-8^{+}$cells (see Results). In all cases, the scoring of stained cluster areas was performed by two independent researchers who agreed on a final score.

\section{Analysis of Hyperplastic Foci in Prostate Lobes}

Hematoxylin and eosin-colored prostate slides from 20month-old $P b-P R L$ mice were used to analyze hyperplasia in prostate lobes of $P b-P R L$ mice. At this age, $P b-P R L$ prostate slides display approximately 10 severely disorganized hyperplastic epithelial areas (including PIN lesions). The 10 most altered epithelial regions in tissue slides of five mice were chosen (total $n=50$ ) regardless of the prostate lobe in which they appeared. Next, the prevalence of these hyperplastic foci per lobe was determined by sorting them according to their lobe of origin.

\section{Determination of Proliferation Index of Prostate Lobes}

Digital images of prostate slides stained by IHC for Ki-67 were obtained from six $P b-P R L$ and three WT mice (approximately 5000 and 1000 total nuclei counted, respectively, per mouse lobe). Counts of positive and negative nuclei in each image were performed by ImageJ version 1.46r (http://imagej.nih gov/ij). Results were expressed as: proliferation index $=$ positive nuclei/total nuclei counted.

\section{Acute PRL Stimulation}

Recombinant human PRL (produced in house) or vehicle (saline solution) was injected subcutaneously into WT mice. After 1 hour, prostate lobes were dissected from each mouse and frozen for protein assays (see above).

\section{RNA Expression}

For RNA extraction, frozen prostate lobes were homogenized with mortar and pestle and processed with an RNeasy Mini kit (Qiagen, Germantown, MD) according to the manufacturer's instructions. Isolated RNA concentrations were measured in a NanoDrop spectrophotometer (Thermo Fisher Scientific), and reverse transcription was performed with the SuperScript FirstStrand Synthesis System for RT-PCR (Life Technologies). For quantitative PCR, SYBR Green PCR Master Mix was used, and reactions were run in an Applied Biosystems 7300 Real-Time 
PCR System (Life Technologies). RNA expression of PRL receptor (PRLR) isoforms was evaluated with previously reported isoform-specific PRLR primers. ${ }^{15}$ Results were normalized to cyclophilin A expression (forward primer, 5' CAGGTCCTGGCATCTTGTCC-3'; reverse primer, 5'-TTGCTGGTCTTGCCATTCCT-3').

\section{Prostate Subpopulation Sorting}

The procedures for prostate cell isolation and sorting were performed according to a published protocol. ${ }^{16}$ In brief, cells were isolated from freshly dissected WT and $P b-P R L$ mouse prostates by tissue mincing, collagenase digestion, treatment with trypsin and DNase, followed by dissociation with syringe and needle and passing through a nylon mesh. Cell suspensions from both genotypes were subjected to differential centrifugation by using Histopaque-1119 (Sigma-Aldrich, St. Louis, MO) to reduce the prostate secretion contents (high in $P b-P R L$ prostates). Isolated cells were then stained for fluorescence-activated cell sorting (FACS) in ice for $20 \mathrm{mi}-$ nutes. Antibodies used for FACS included fluorescein isothiocyanate-coupled lineage (Lin) antibodies (anti-CD31, CD45 and Ter119, 11-0311-85, 11-0451-85, 11-05921-85, respectively); phosphatidylethanolamine-coupled anti-CD49f (integrin alpha-6; 12-0495-83); and allophycocyanin-coupled anti-Sca-1 (lymphocyte antigen 6A-2/6E-1; 17-5981-82) all from eBioscience (San Diego, CA). Dead cells were colored with SYTOX Blue (Life Technologies). Cell sorting was performed on a BD FACSAria I (BD Biosciences). Sorted cells were collected in Dulbecco's modified Eagle's medium, supplemented with $50 \%$ bovine fetal serum, glutamine, and penicillin-streptomycin.

\section{Sphere-Generation and Colony Assays}

For the sphere-generation assay, sorted cells were washed, resuspended in PrEGM (Lonza, Basel, Switzerland), mixed with Matrigel (BD Biosciences) in a 40:60 ratio, and plated around the rim of the wells of a 12-well plate (5000 to 10,000 cells per well). Every 3 days, half the volume of PrEGM was changed. Sphere generation was determined at day 7. For the colony assay, sorted cells were washed and seeded in Matrigelcoated 12-well plates $(10,000$ cells per well) in PrEGM. Following a published protocol, ${ }^{16}$ colonies were grown for 7 days and then stimulated with $0.01 \mu \mathrm{mol} / \mathrm{L} 5 \alpha$-dihydrotestosterone (Sigma-Aldrich) for 7 more days. Medium was changed every 2 to 3 days. Colonies were then fixed in cold acetone, blocked, and stained by IHC and IF as explained above.

\section{MTT Staining and Sphere Analysis}

Spheres in three-dimensional culture were stained with MTT by addition to the culture medium of $100 \mu \mathrm{L} /$ well of a $5-\mathrm{mg} / \mathrm{mL}$ dilution. After 2 hours, complete wells with stained spheres were photographed on a white light transilluminator by using a digital camera. Digital images were analyzed by ImageJ version $1.46 \mathrm{r}$ to measure the number of particles (spheres) and their cross-sectional area. Average diameter $(\mu \mathrm{m})$ was calculated for each sphere according to its cross-sectional area, using the formula

$$
\left[\text { diameter }=2 \times(\text { cross-sectional area } / \Pi)^{1 / 2}\right] .
$$

\section{Cytospin of Sorted Cell Populations}

Sorted cell populations were washed and fixed in $4 \%$ paraformaldehyde. Fixed cells were then resuspended in phosphate-buffered saline and spun in a Shandon Cytospin 2 Cytocentrifuge (Thermo Scientific). Slides with cells were left to dry at room temperature. For IF, the protocol was the same as described for tissue slides, except for the addition of a permeabilization step in Tris-buffered saline $+0.1 \%$ Triton X-100 after antigen retrieval.

\section{Statistical Analysis}

The specific statistical tests performed are described for all results reported. In summary, analysis of variance (ANOVA) tests were used to evaluate differences among three or more groups. Depending on the number of factors tested, one- or two-way ANOVAs were used (one or two factors tested, respectively). When prostate lobe samples issued from the same mice were compared in the analysis, a repeated measures test was performed. Post hoc multiple comparisons were performed by Bonferroni's test. A Pearson's correlation test was used to evaluate the association between mean values of Stat 5 activation and of basal cell cluster occurrence per lobe. Contingency analyses were performed on frequencies of staining scores for IHC/IF of basal cell cluster areas by a two-tailed Fischer's exact test. Prostasphere numbers were compared with a paired $t$-test and prostasphere size distributions by a $\chi^{2}$ test for trend. Differences in proliferation status among different cell subpopulations also were tested by $\chi^{2}$. A value of $P<0.05$ was used as significance cutoff for all tests. All analyses were performed with GraphPad Prism version 6.00 for Windows (GraphPad Software, San Diego CA).

\section{Results}

Basal Cell Clusters Are More Prevalent in Dorsal than Ventral Lobes of $P b-P R L$ Prostates

Our previous results that involved dorsal lobes of $P b-P R L$ mice indicated that PRL overexpression induced marked proliferation of epithelial cells, including notably the basal/ stem cell compartment. ${ }^{3}$ As stated in the Introduction, these cells frequently displayed abnormal morphology and formed clusters. To establish whether PRL induced the appearance of basal cell clusters to similar degrees in all prostate lobes, we compared the number of clusters in ventral, lateral, and dorsal lobes of $P b-P R L$ and WT mice. 
Basal cell clusters in the different lobes were counted after staining of tissue slides for the basal cell marker CK-5. This marker was chosen over p63 after comparison of the two stainings, because CK-5 stained the cytoplasm of basal cells (as opposed to nuclear p63 staining) and thus allowed for detection of basal cells even when their nucleus was outside the plane of the tissue slice observed. Examples of basal cell clusters are shown in Figure 1A. Prostates of both genotypes (WT and $P b-P R L$ ) displayed clusters in proximal regions of the various lobes (where basal cells are usually more densely disposed). In contrast, intermediate and distal
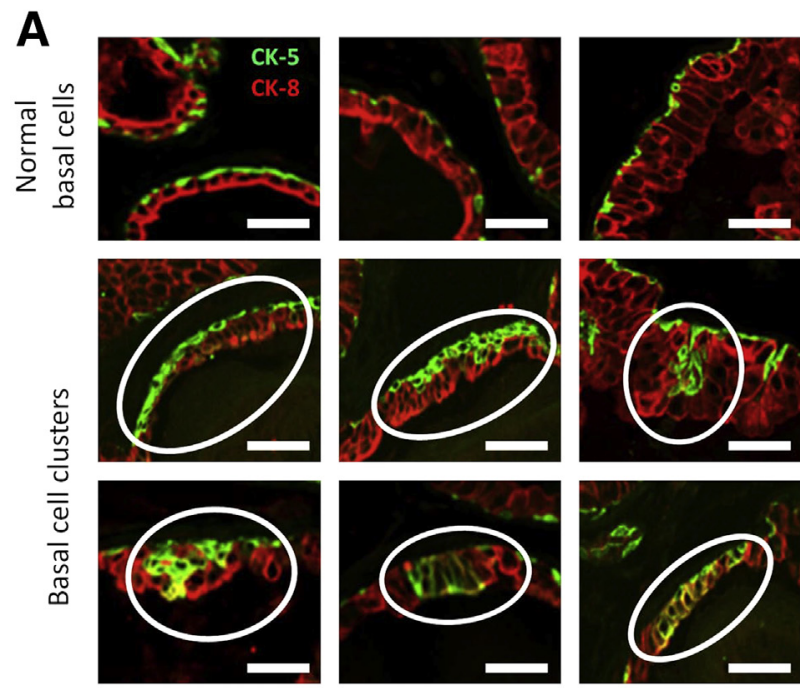

B

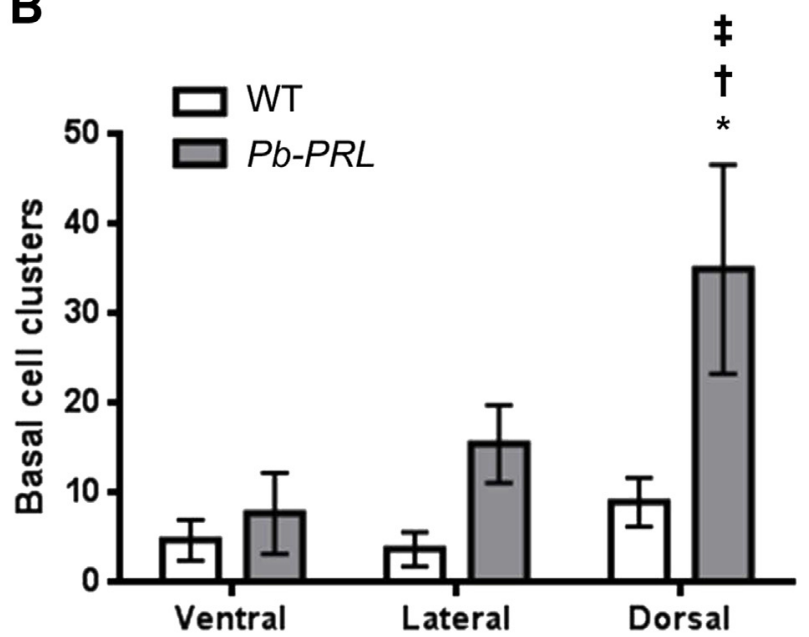

Figure 1 Basal cell clusters in WT and Pb-PRL prostate lobes. A: IF images of $P b-P R L$ prostate slides stained for basal (CK-5) and luminal (CK-8) cytokeratins. Three example images of normal basal cells are shown on the top row; examples of basal cell clusters are highlighted in white circles in the images of middle and bottom rows. Basal cell clusters containing double positive $\mathrm{CK}-5^{+} \mathrm{CK}-8^{+}$cells (yellow) are shown in the images of the bottom row. B: Basal cell clusters were counted in prostate lobes of WT and $P b$-PRL mice. Statistical significance was evaluated by two-way repeated measures ANOVA and Bonferroni's multiple comparisons test. All data are expressed as means \pm SD. $n=4$ mice per group (B). ${ }^{*} P<0.05$ versus WT; ${ }^{\dagger} P<0.01$ versus ventral lobe; ${ }^{\ddagger} P<0.05$ versus lateral lobe. Scale bar $=50$ $\mu \mathrm{m}(\mathbf{A})$. Original magnification: $\times 10(\mathbf{A})$. regions contained clusters mostly in $P b-P R L$ prostates. As expected, our results indicated a significant increase in the number of basal cell clusters found in $P b-P R L$ compared with WT prostates. Notably, this increase was particularly marked in dorsal lobes of $P b-P R L$ prostates, where basal cell clusters were more numerous than in lateral and ventral lobes (Figure 1B). These data suggested that the effects of PRL overexpression in the $P b-P R L$ mouse prostate were lobe specific.

\section{Stat5 Activation Significantly Correlates with Basal Cell Cluster Occurrence in Prostate Lobes}

Given the disparity detected in the number of basal cell clusters counted in prostate lobes of $P b-P R L$ mice, we hypothesized that the occurrence of clusters in each lobe might reflect its respective degree of PRL signaling activation. Our previous results indicated that PRL signaled mainly via p-Stat5 in the dorsal prostate. ${ }^{3}$ Thus, we measured Stat5 activation in individual lobes of $P b-P R L$ and WT prostates. Results of Western blot analysis found faint levels of p-Stat5 in the ventral lobe of $P b-P R L$ animals, which were not significantly different from WT levels (Figure 2, A and B). In contrast, lateral and dorsal lobes displayed significantly enhanced Stat5 activation compared with WT lobes. These densitometric quantifications were fully confirmed by semiquantitative AlphaScreen SureFire homogeneous assays (Figure 2C). These data indicated that Stat5 activation was not uniform among prostate lobes of $P b-P R L$ mice.

To determine whether other PRL-stimulated pathways (Jak2/Stat3; phosphatidylinositol 3-kinase/Akt; mitogenactivated protein kinase) were activated specifically in the ventral lobe, we performed Western blot analyses of $\mathrm{p}-\mathrm{Stat} 3$, p-Akt, and p-Erk1/2 (Figure 2, A and D-F). None of these pathways were activated in the ventral lobe of $P b-P R L$ mice compared with WT mice. The lateral and dorsal lobes did not indicate activation either, consistent with our previous findings in dorsal prostate of $P b-P R L$ mice. ${ }^{3}$ In summary, these results indicated that Stat5 was the only canonical PRLR signaling pathway triggered by local PRL in $P b-P R L$ prostates. Importantly, the level of activation of this pathway was significantly correlated with the prevalence of basal cell clusters in each lobe (Figure $2 \mathrm{H}$ ).

PRL Insensitivity of $P b-P R L$ Ventral Prostates Is Due to Decreased Levels of PRLR

Low PRL signaling in ventral lobes of $P b-P R L$ mice might be due to poor transgene expression that results in low PRL levels in this lobe. Even though expression of the PRL transgene had been verified at the mRNA level in all lobes, including ventral prostate, ${ }^{14}$ PRL protein levels had not been reported. Thus, we compared PRL levels in individual lobes by Western blot analysis (Figure 2, A and G). As expected, PRL levels were significantly higher in all lobes of $P b-P R L$ mice than in WT mice (where PRL was 

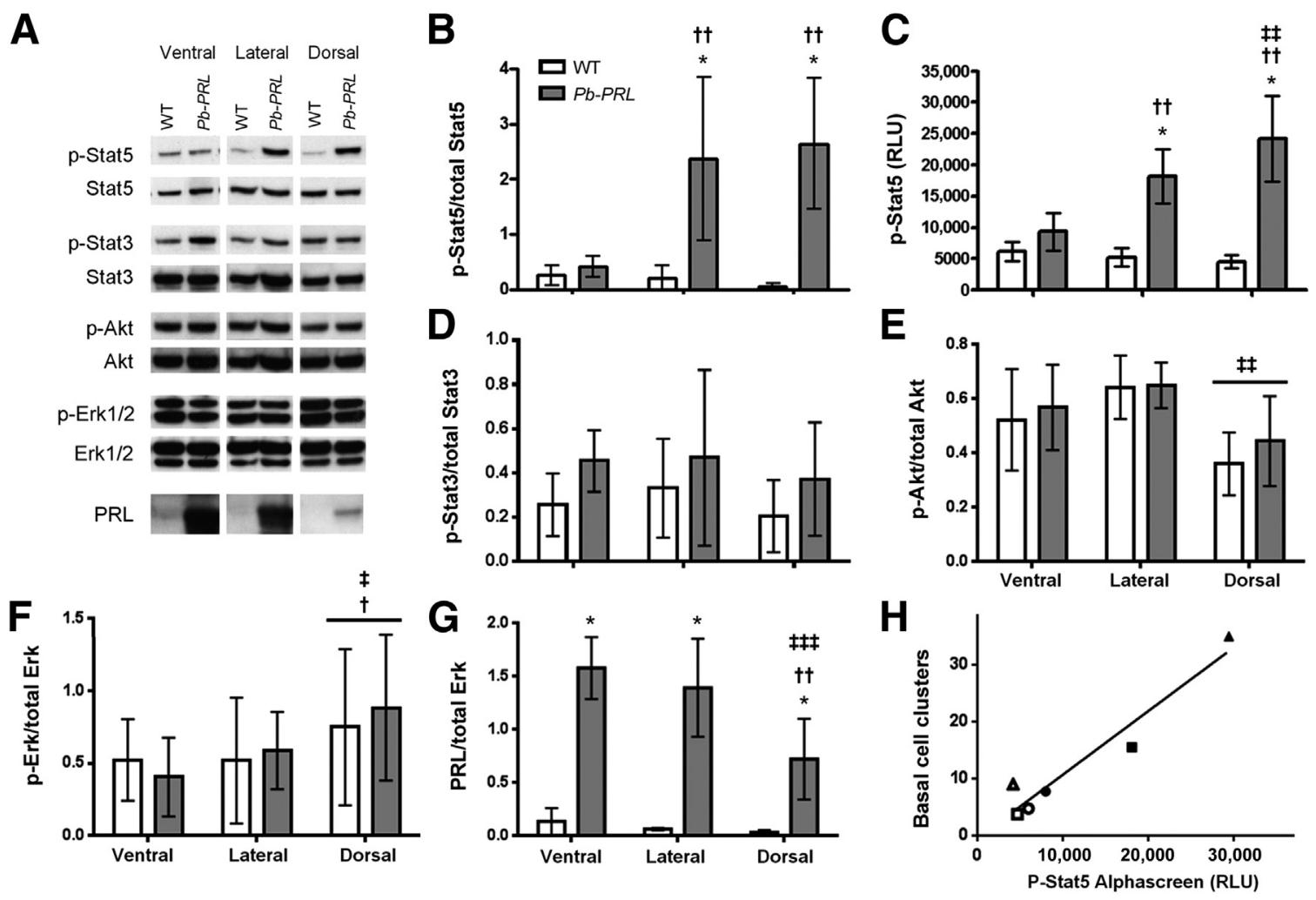

Figure 2 Activation of canonical PRLR signaling pathways in WT and Pb-PRL ventral, lateral, and dorsal prostate lobes. A: Representative Western blot images per genotype. B: Quantification of p-Stat5 blot results normalized to total Stat5 levels. C: Results of semiquantitative AlphaScreen SureFire assay for p-Stat5 levels in WT and Pb-PRL mice. D-G: Quantification of results from Western blot analysis for p-Stat3, p-Akt, p-Erk1/2 (normalized to total Stat3, Akt, and Erk1/2 levels, respectively) and PRL levels (normalized to total Erk1/2 levels). H: Correlation between mean counts of basal cell clusters and mean levels of p-Stat5 measured by semiquantitative AlphaScreen SureFire assay in each prostate lobe. WT and Pb-PRL mouse values are represented with white and black symbols, respectively (circles, ventral lobes; squares, lateral lobes; triangles, dorsal lobes). Data for all bar graphs are expressed as means \pm SD. $n=6$ mice per genotype (B); $n=5$ WT mice (C); $n=12 P b$-PRL mice (C); $n=6$ mice per genotype (D-G). ${ }^{\star} P<0.001$ versus WT; ${ }^{\dagger} P<0.01,{ }^{\dagger \dagger} P<0.001$ versus ventral lobe; ${ }^{\ddagger} P<0.05,{ }^{\ddagger \ddagger} P<0.01,{ }^{\ddagger \ddagger \ddagger} P<0.001$ versus lateral lobe by two-way repeated measures ANOVA and Bonferroni's multiple comparisons test (B-G). Pearson correlation test, $P=0.002 ; r^{2}=0.93(\mathbf{H})$.

essentially undetectable). Among $P b-P R L$ lobes, levels of PRL were significantly higher in ventral and lateral lobes than in dorsal lobes (Figure 2, A and G). Similar results were observed by IHC of $P b-P R L$ prostate slides (Supplemental Figure S1). The general lack of PRL signaling activation in the ventral lobe despite the presence of PRL suggested a certain degree of insensitivity of the $P b$ $P R L$ ventral prostate to this hormone.

In an effort to understand this insensitivity to PRL, we tested the responsiveness of WT prostate lobes to acute PRL stimulation. WT mice were injected with $50 \mu \mathrm{g}$ of PRL, and prostates were collected after approximately 60 minutes. Significant Stat5 activation was observed in all lobes by Western blot analysis (Figure 3, A and B) and by AlphaScreen SureFire assay (Figure 3C). These data indicated that PRL normally triggered Stat 5 signaling in the ventral lobe, which suggested that the lack of Stat5 activation observed in ventral lobes of $P b-P R L$ mice (Figure 2, $\mathrm{A}-\mathrm{C}$ ) might be the result of negative regulatory mechanisms progressively established on chronic PRL stimulation.

We then explored the possibility that the insensitivity to PRL in the ventral lobe of $P b-P R L$ mice could be due to the alteration of PRLR expression. The mouse PRLR is expressed as four different isoforms (Long/L, Short/S1, S2, and S3), which result from alternative splicing. ${ }^{17}$ Of note, short isoforms are known to exert dominant negative effects on the activation of the Jak2/Stat5 pathway by the long isoform ${ }^{18}$; therefore, not only the absolute expression level of each isoform but also their ratio needed to be assessed. The expression profiles of PRLR isoforms in ventral and dorsal lobes were the same in WT and $P b-P R L$ prostates; that is, the $\mathrm{L}$ isoform was the most prevalent, followed by markedly lower levels of the S3 isoform and negligible levels of the S2 isoform; S1 was not detected (Supplemental Figure S2). We then compared the levels of the L and S3 isoforms in the ventral and dorsal lobes of WT and $P b-P R L$ mice. Analysis of the ventral lobe indicated significantly lower expression of both $\mathrm{L}$ and $\mathrm{S} 3$ isoforms in $P b-P R L$ prostates (Figure 3, D and E). Analysis of the dorsal lobe indicated a slight decrease, but the differences between genotypes were not significant. Moreover, the ratio between the $\mathrm{S} 3$ and $\mathrm{L}$ isoforms of PRLR displayed no differences between genotypes (Figure 3F). These results indicated that the PRL insensitivity of $P b-P R L$ ventral prostate was due, at 




D





$\mathbf{E}$

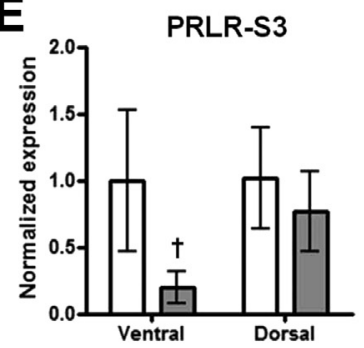

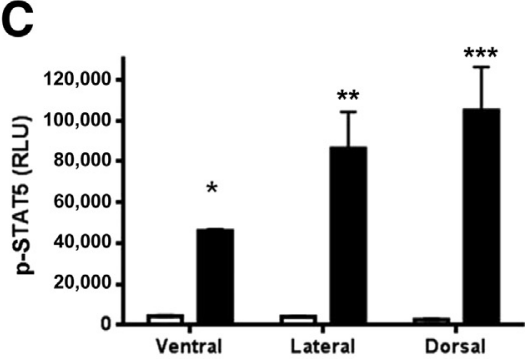

$\mathbf{F}$

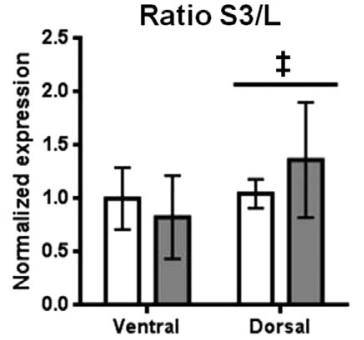

Figure 3 Response to acute PRL stimulation in prostate lobes of WT mice and PRLR levels in prostate lobes of WT and Pb-PRL mice. A: Representative image of results from Western blot analysis for Stat5 activation after subcutaneous injection of vehicle or $50 \mu \mathrm{g}$ of PRL. B: Quantification of p-Stat5 blotting results normalized to total Stat5 levels. C: Results of semiquantitative AlphaScreen SureFire assay for p-Stat5 levels. D-F: mRNA expression levels of long L (D) and short3 S3 (E) PRLR isoforms and S3/L ratios (F) in dorsal and ventral lobes of WT and Pb-PRL mice. All data are represented relative to WT ventral expression levels after normalization to cyclophilin A. Data are expressed as means \pm SD. $n=4$ mice per group (B); $n=3$ mice per group (C); $n=5$ mice per group (D-F). ${ }^{*} P<0.05,{ }^{* *} P<0.01,{ }^{* *}{ }^{*} P<0.001$ versus vehicle treatment (B and $\mathbf{C}$ ) $;{ }^{\dagger} P<0.01,{ }^{\dagger \dagger} P<0.001$ versus WT; ${ }^{\ddagger} P<0.01$ versus ventral lobe by two-way repeated measures ANOVA and Bonferroni's multiple comparisons test $(\mathbf{D}-\mathbf{F})$. D, dorsal; $L$, lateral; $V$, ventral.

least in part, to a decrease in PRLR-L expression in this lobe and not to an increased dominant negative effect of the S3 isoform on Stat5 signaling.

All together, the data reported here suggest that i) Stat5 was the main signaling molecule activated by PRL in $P b$ $P R L$ prostates, ii) Stat5 activation was associated with the occurrence of basal cell clusters in prostate lobes of $P b-P R L$ mice, and iii) lobe-related differences in the prevalence of PRL effects in $P b-P R L$ prostates were presumably due to dissimilar levels of PRLR expression.

\section{Epithelial Hyperplasia Is More Prevalent in Dorsal than Ventral Lobes of Pb-PRL Mice Despite Similar Proliferation Rates and Weight Gain in all Lobes}

Previously described histological alterations at 3 months of age in the $P b-P R L$ prostate epithelium included distended ducts, flattened epithelium, and regular zones of hyperplasia with or without atypical features. ${ }^{14}$ The observation that PRL signaling and basal cell cluster occurrence were considerably reduced in the ventral lobe of $P b-P R L$ mice prompted us to analyze whether these previously described histological alterations were also less prevalent in the ventral prostate. To evaluate this hypothesis, we analyzed tissue slides of ventral, lateral, and dorsal lobes of WT and $P b-P R L$ prostates.

Our analysis of mice older than 6 months of age confirmed the presence of duct distention and hyperplasia in the $P b-P R L$ epithelium but indicated that these alterations were present to different degrees in each lobe. In fact, the distention of ducts was generally more pronounced in ventral and lateral lobes, whereas hyperplastic areas, including PIN lesions, were particularly more prevalent in the dorsal lobe, followed by the lateral lobe (Figure 4A). In an effort to quantify this phenomenon, hyperplastic foci with or without atypical features were analyzed in prostate tissue slides of $P b-P R L$ mice (Figure 4B). This particular analysis was performed on slides of 20-month-old animals which present extensive histological alterations. Our results found that the prevalence of epithelial hyperplasia was significantly higher in dorsal lobes than in ventral lobes (Figure 4B).

In addition, the degree of epithelial proliferation in each prostate lobe was calculated with Ki-67 staining and normalizing to the total number of nuclei in that area (proliferation index). The results found as much epithelial proliferation in the ventral lobe as in the dorsal lobe of $\mathrm{Pb}$ PRL mice (Figure 4, C and D). Consistent with similar proliferation rates, wet weights of individual prostate lobes found a significant enlargement in all lobes of $P b-P R L$ mice compared with WT mice (Figure 4, E-G). The enlargement of all lobes in $P b-P R L$ prostates increased as mice got older (approximately 20 months of age; data not shown) and was consistent with previous reports. ${ }^{14}$ The fact that all prostate lobes of $P b-P R L$ mice displayed similarly increased weights and proliferation rates indicated that these variables do not depend exclusively on Stat5 activation. In contrast, the higher prevalence of hyperplastic foci in dorsal lobes of $\mathrm{Pb}$ $P R L$ prostates suggested that PRL/Stat5 signaling may play a relevant role in the development of these foci. 


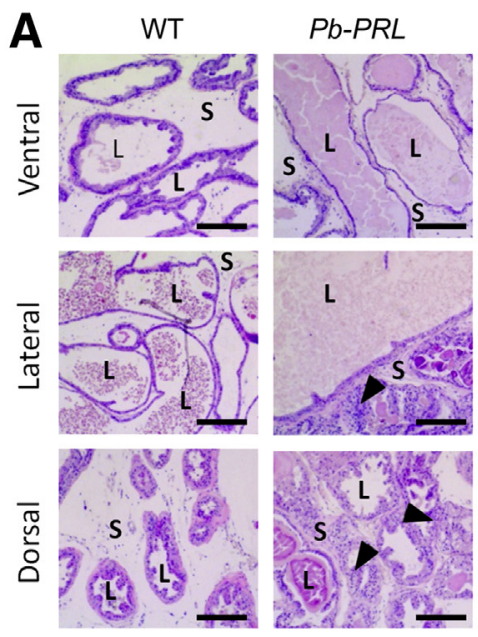

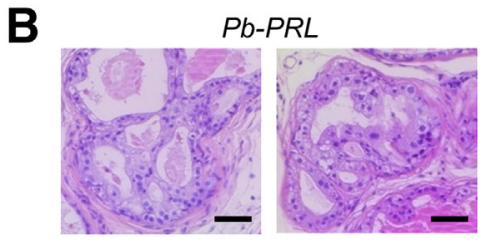

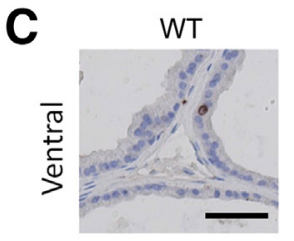

$P b-P R L$


G
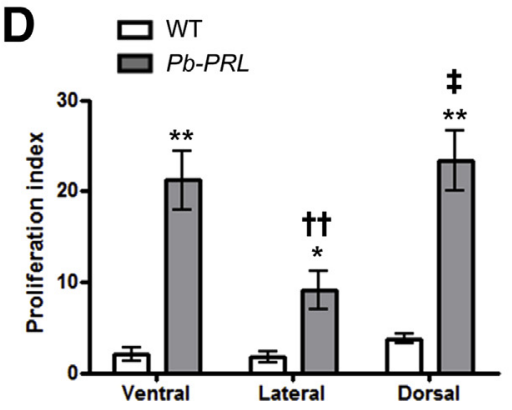

E

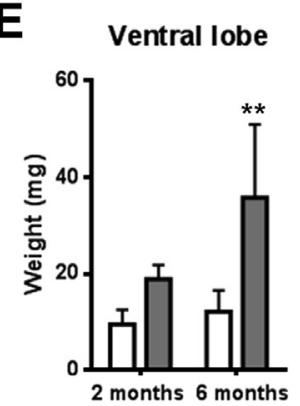

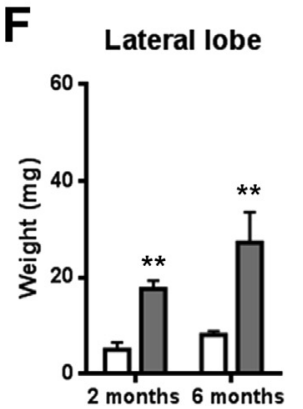

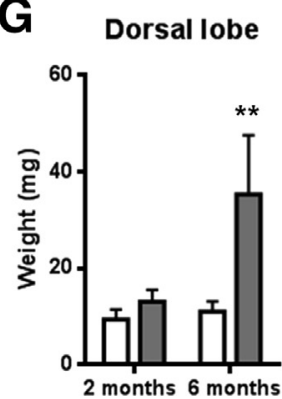

Figure 4 Histology, hyperplastic foci, proliferation index, and weight of WT and $P b$-PRL prostate lobes. A: Representative hematoxylin and eosin images of tissue histology in each lobe. Pb-PRL slides displayed marked gland distention in ventral and lateral lobes, whereas hyperplastic foci (arrowheads) were more prevalent in dorsal lobes, followed by lateral lobes. B: Hyperplastic areas were counted in prostate lobes of $P b-P R L$ mice at 20 months (two examples shown). C: Representative IHC images of Ki-67 staining used for quantification of proliferation in WT and $P b-P R L$ prostate lobes. D: Quantification of Ki-67 staining (proliferation index) in WT and Pb-PRL prostate lobes. E-G: Ventral (E), lateral (F), and dorsal (G) prostate lobe weights in WT and $P b-P R L$ mice at 2 months and 6 months. Data are expressed as means \pm SD. $n=50$ areas in 5 mice (B); $n=3$ WT prostate lobes (D); 6 Pb-PRL mice (D); $n=5$ WT mice at 2 months and $8 \mathrm{~Pb}$-PRL mice at 2 months $(\mathbf{E}-\mathbf{G}) ; n=5$ WT mice at 6 months and $6 P b-P R L$ mice at 6 months (E-G). ${ }^{*} P<0.01,{ }^{* *} P<0.001$ versus WT; ${ }^{\dagger} P<0.01$, ${ }^{\dagger \dagger} P<0.001$ versus ventral lobe; ${ }^{\ddagger} P<0.001$ versus lateral lobe by one-way ANOVA (B), two-way ANOVA (D), and two-way repeated measures ANOVA (D-G), all followed by Bonferroni's multiple comparisons tests. Scale bars: $200 \mu \mathrm{m}(\mathbf{A}) ; 50 \mu \mathrm{m}$ (B and C). Original magnification: $\times 10(\mathbf{A}) ; \times 40$ (B and C). L, lumen; S, stroma.

\section{Basal Cell Cluster Areas in Pb-PRL Prostates Display Increased Nuclear Stat5, Sca-1 Staining, and Frequency of Intermediate $\mathrm{CK}-5^{+} \mathrm{CK}-8^{+}$Cells}

Our results above indicated that prostate lobes with enhanced Stat5 activation displayed a higher prevalence of basal cell clusters. In an effort to characterize this association further, we specifically analyzed basal cell cluster areas in the prostate epithelium for the presence of nuclear Stat5 within the cluster area. Associations between these basal cell clusters and markers of proliferation (Ki-67) and differentiation (CK-5, CK-8, Sca-1) were also evaluated. Sca-1 is a stem/progenitor marker in the prostate epithelium, ${ }^{19}$ which we previously reported to be increased in the $P b-P R L$ dorsal prostate. ${ }^{3}$ Our analysis involved a series of IHC/IF stainings performed on prostate tissue slides of $P b-P R L$ and WT mice. Epithelial areas surrounding basal cell clusters (including the immediate neighboring luminal epithelial cells) were identified by IF (Figure 1A) and were analyzed on serial slides stained by IHC for Ki-67, Stat5, and Sca-1. Scales of staining scores from 0 to
5 were established for each of Sca-1, Ki-67, and nuclear Stat5 stainings, according to the percentage of positive cells in the area and the staining intensity (Supplemental Figure S3). Given that Sca-1 staining was frequently present in the lumen (secretions) and the stroma, special care was taken to count as positive only the epithelial membrane staining. In addition, the presence of double-positive CK- $5^{+} \mathrm{CK}-8^{+}$(intermediate) cells in each cluster area was evaluated by IF and scored as 0 , no (absence) and 1, yes (presence). Scoring of all stained cluster areas was performed by two independent researchers who agreed on a final score.

To increase the accuracy of our qualitative scoring method, the scores were grouped into two categories ( 0 to 2 as low and 3 to 5 as high scores). We then compared the frequencies of detection for high versus low stainings between cluster areas in WT and $P b-P R L$ mice. High nuclear Stat5, high Sca-1 staining, and presence of intermediate CK- $5^{+} \mathrm{CK}-8^{+}$cells were more prevalent within basal cell cluster areas in $P b-P R L$ prostate tissue than in WT prostate tissue (Figure 5, A-C). These data suggested that epithelial areas baring basal cell 
A



C



$\mathbf{F}$ Ki-67 vs Double CK- $5^{+}$CK- $8^{+}$

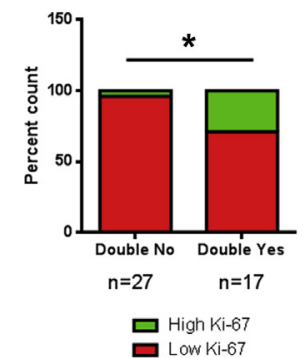

B


$\mathbf{D}_{\mathrm{s}+1}$

Sca- 1 vs Double CK- $5^{+}$CK- $8^{+}$



$\mathbf{E}$

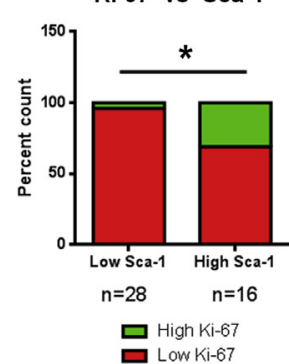

Figure 5 Analysis of basal cell clusters in WT and $P b$-PRL mice. A-C: Frequency distributions for staining scores in basal cell cluster areas of each genotype: high versus low nuclear Stat5 (A) and Sca-1 (B) staining, and yes versus no for the presence of double positive $\mathrm{CK}-5^{+} \mathrm{CK}-8^{+}$cells $(\mathbf{C})$. Representative images of basal cell cluster areas that showed high versus low or yes versus no scores for each staining are shown on the right of each graph; together with corresponding CK-5/CK-8 IF images. D-F: Distribution of staining results detected in basal cell cluster areas of Pb-PRL mice for Sca-1 when double positive CK$5^{+} \mathrm{CK}-8^{+}$cells were present (yes) versus absent (no) (D), for Ki-67 when Sca- 1 was high versus low (E), and when double $\mathrm{CK}-5^{+} \mathrm{CK}-8^{+}$cells were yes versus no (F). G-H: Representative images of serial slides for two basal cell cluster areas displaying both presence of double positive CK- $5^{+}$CK- $8^{+}$cells (IF staining) and high Sca-1 and Ki-67 scores (IHC staining). Basal cell cluster areas analyzed are encircled by dashed lines; arrows point to one or more double positive CK- $5^{+}$CK- $-8^{+}$cells. Sample sizes $(n)$ are indicated on each graph. ${ }^{*} P<0.05,{ }^{*} P<0.01$, and ${ }^{* * *} P<0.001$ by two-tailed Fischer's exact test $(\mathbf{A}-\mathbf{F})$. Scale bar: $25 \mu \mathrm{m}(\mathbf{A}-\mathbf{C}, \mathbf{G}$, and $\mathbf{H})$. Original magnification: $\times 40(\mathbf{A}-\mathbf{C}, \mathbf{G}$, and $\mathbf{H}$, IHC images); $\times 10(\mathbf{A}-\mathbf{C}, \mathbf{G}$, and $\mathbf{H}$, IF images).

clusters are not only more numerous in $P b-P R L$ mice than in WT mice but that their signaling and differentiation status are different from occasional basal cell clusters detected in WT epithelia. Importantly, the correlation observed in Figure $2 \mathrm{H}$ between Stat5 activation and presence of basal cell clusters found in prostate lobes was here confirmed at the cellular level within basal cell cluster areas. However, as previously reported, ${ }^{3}$ nuclear Stat5 staining was localized to the luminal layer and not the basal layer of the prostate epithelium. These results suggested that paracrine mechanisms between the two cell types may be involved in the formation of basal cell clusters.

However, no differences were observed among $P b-P R L$ prostate lobes in terms of staining scores within basal cell cluster areas (data not shown). These results suggested that the degree of PRL signaling activation (ie, p-Stat5) might dictate the frequency of development of basal cell clusters (ventral $<$ dorsal lobe), but once generated, these clusters displayed a similar phenotype regardless of the lobe in which they originated.

High Sca-1 Staining Associates with High Proliferation and Presence of Intermediate $\mathrm{CK}-5^{+} \mathrm{CK}-8^{+}$Cells in $P b-P R L$ Basal Cell Cluster Areas

To test for possible associations among the different stainings detected in basal cell cluster areas of $P b-P R L$ mice, we compared high versus low (or yes versus no) frequencies by 
contingency analyses. No such analyses were possible in WT mice, given that few ( $n=1$ or 2$)$ cluster areas displayed high/yes scores for the tested stainings, thus rendering the data too unbalanced. In $P b-P R L$ mice, Sca-1 staining scores were higher in cluster areas with intermediate $\mathrm{CK}-5^{+} \mathrm{CK}-8^{+}$cells (Figure 5D). In addition, high Sca-1 staining and presence of intermediate $\mathrm{CK}-5^{+} \mathrm{CK}-8^{+}$ cells associated with high proliferation scores (Figure 5, E and $\mathrm{F}$ ). Figure 5, G and $\mathrm{H}$, shows representative $P b-P R L$ cluster areas that exhibit double $\mathrm{CK}-5^{+} \mathrm{CK}-8^{+}$cells, high Sca-1, and high Ki-67 staining.

\section{FACS Analysis of $P b$-PRL Prostates Indicates the Presence of Two Sca- $1^{+}$Epithelial Cell Subpopulations}

The amplification of basal/stem cells detected in $P b-P R L$ prostates might be at the origin of prostate tumor development in this model, especially given their reported role as cells of origin for prostate cancer. ${ }^{7}$ We used a complementary approach to further characterize the amplification of basal/stem cells in $P b-P R L$ mice by using a published FACS protocol. ${ }^{16}$ Isolated prostate cells can be sorted into basal/ stem, luminal, and stromal subpopulations. Given the small size of prostate lobes, and the need to pool several mice per FACS experiment, these analyses were performed on whole prostates rather than on individual lobes.

Previous reports ${ }^{8}$ and our results found that WT mice mainly display the three mentioned cell subpopulations when resolved with Lin/Sca-1/CD49f surface staining (Figure 6A). $\mathrm{Lin}^{-}$cells consist of three subpopulations that can be observed as Sca- $1^{-}$CD $49 \mathrm{f}^{\text {med }}$ (luminal), Sca- $1^{+}$CD $49 \mathrm{f}^{\text {high }}$ (LSC, basal/ stem, here called LSC-high), and Sca- $1^{+} \mathrm{CD} 49 \mathrm{f}^{-}$(stromal). When $P b-P R L$ prostate cells were analyzed, we observed the expected increase in the basal/stem cell subpopulation (LSChigh) compared with that of WT prostate cells (Figure 6, A and C), consistent with our IHC results. In addition, the $P b-P R L$ mice clearly displayed a fourth subpopulation $\left(\mathrm{Lin}^{-} \mathrm{Sca}-\right.$ $1^{+} \mathrm{CD} 49 \mathrm{f}^{\text {med }}$, LSC-med) (Figure 6, A and B). The appearance of this subpopulation in $P b-P R L$ mice was accompanied by a reciprocal and significant decrease in the $\mathrm{Lin}^{-} \mathrm{Sca}-$ $1^{-} \mathrm{CD} 49 \mathrm{f}^{\text {med }}$ (luminal) cell subpopulation (Figure 6C).

\section{Amplified LSC-Med Cells Display Luminal Characteristics and May Contain Highly Proliferating Progenitors}

We then aimed to characterize the LSC-med subpopulation amplified in $P b-P R L$ prostates. With the use of FACS and stringent sorting gates to avoid contamination (Figure 7A) each subpopulation was collected and subjected to subsequent analyses. To evaluate CK expression, cells were fixed and costained for CK-5 and CK-8 by using IF (Figure 7D). As expected from previous reports, ${ }^{8}$ basal/stem LSC-high cells were mainly $\mathrm{CK}-5^{+}$and luminal cells were mostly $\mathrm{CK}-8^{+}$. Several double $\mathrm{CK}-5^{+} / \mathrm{CK}-8^{+}$(intermediate) cells were present in both subpopulations as well (Figure 7D). The amplified LSC-med subpopulation displayed similar properties as luminal cells, that is, mainly $\mathrm{CK}-8^{+}$cells, with few intermediate cells (Figure 7D). As a control, stromal cells were negative for both CKs.

We also tested the ability of LSC-med cells to generate spheres in three-dimensional culture compared with the

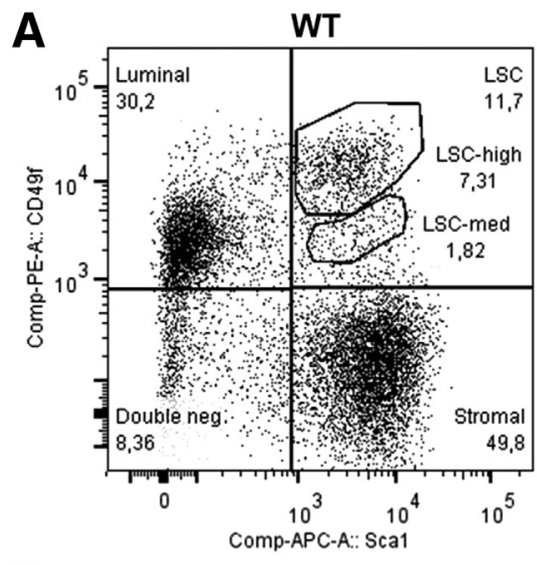

B

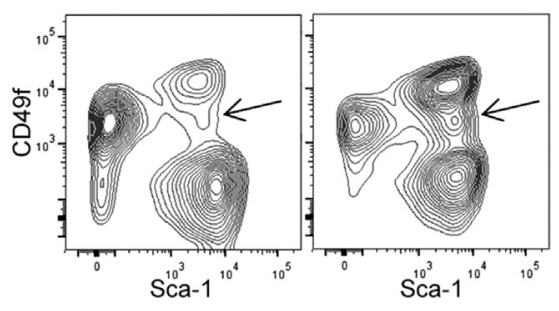

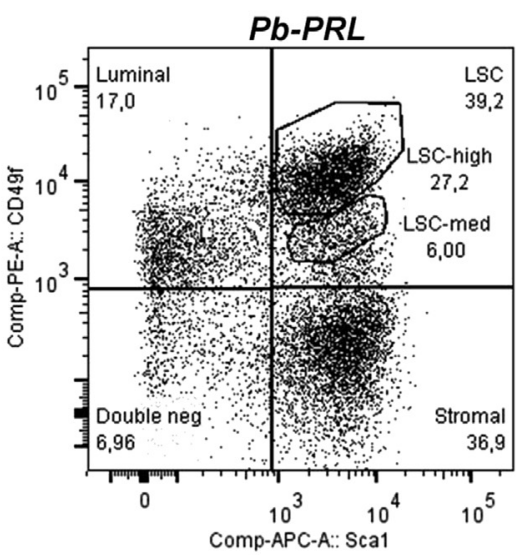

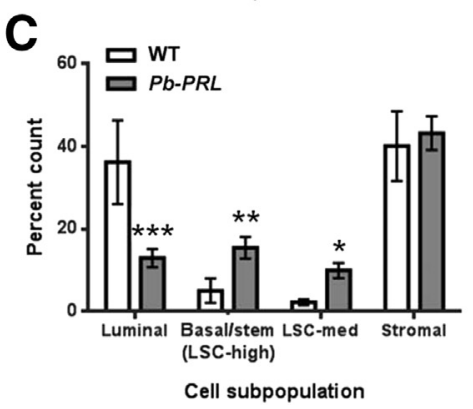

Figure 6 FACS analysis of prostate cell subpopulations in WT and Pb-PRL mice. A: Representative FACS profiles for $W T$ and $P b$-PRL mice showing gated subpopulations. Circled areas show LSC-high and LSC-med subpopulations. Luminal and stromal subpopulations are shown on the top left and bottom right quadrants, respectively. B: Contour plot representation of the same FACS profiles as in $\mathbf{A}$ to illustrate changes in subpopulation percentages, particularly in LSC-med cells (arrows). C: Cell subpopulation percentages measured by FACS in WT and $P b-P R L$ mice. Results include experiments involving mice of ages 6 to 20 months. $n=7$ experiments by genotype, involving $n=3$ to 7 WT and $n=1$ to $4 P b-P R L$ mice of similar ages per experiment (C). ${ }^{*} P<0.05,{ }^{* *} P<0.01$, and ${ }^{* * *} P<0.001$ versus WT by two-way repeated measures ANOVA for experiments paired by age; post hoc comparisons were performed by Bonferroni's multiple comparisons test. 

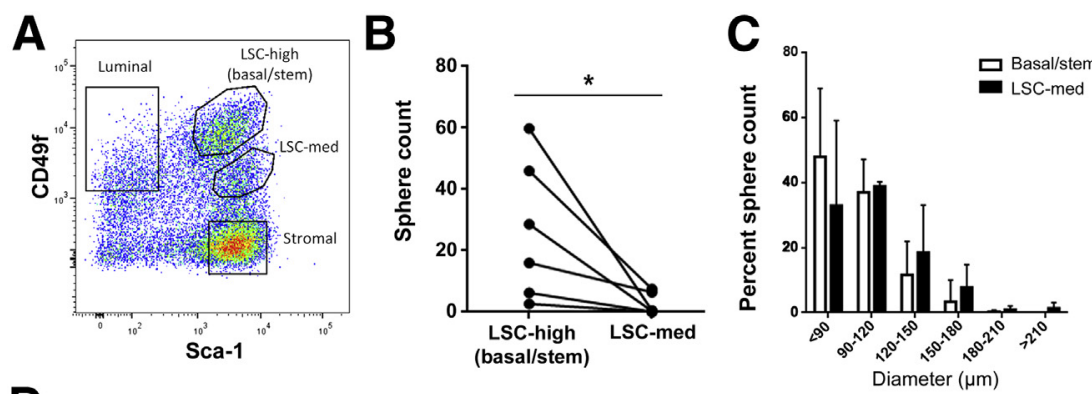

D
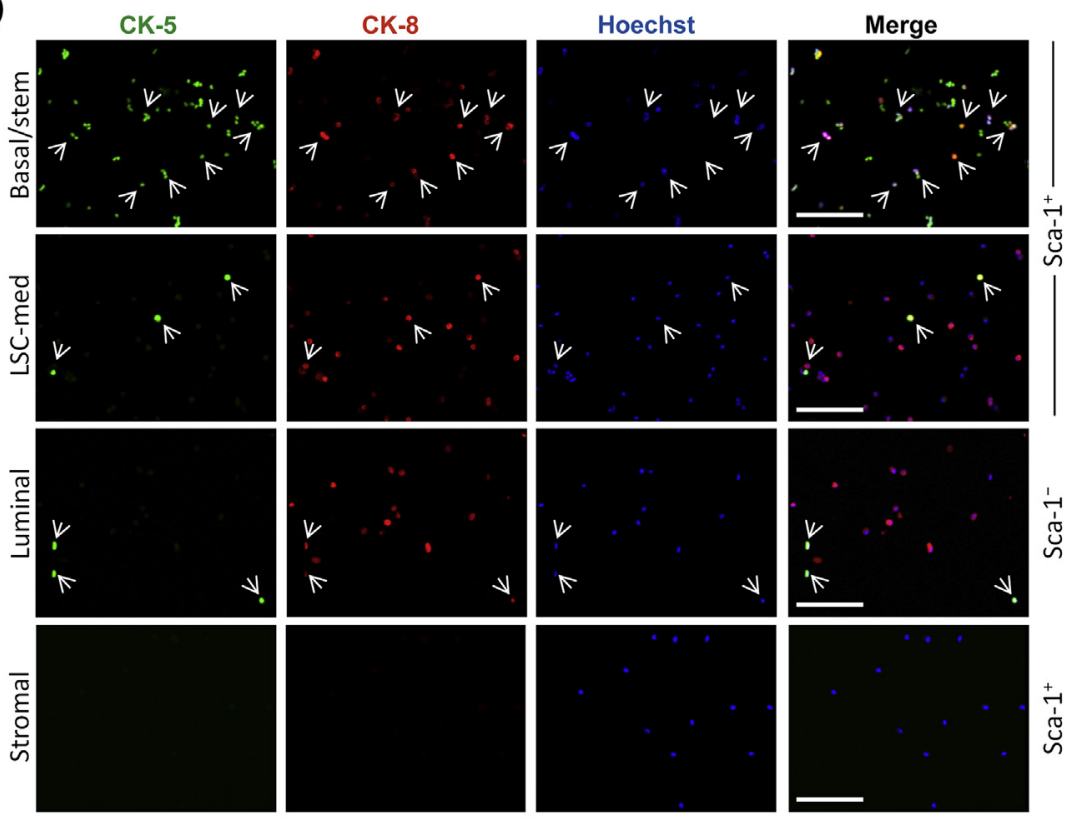

E
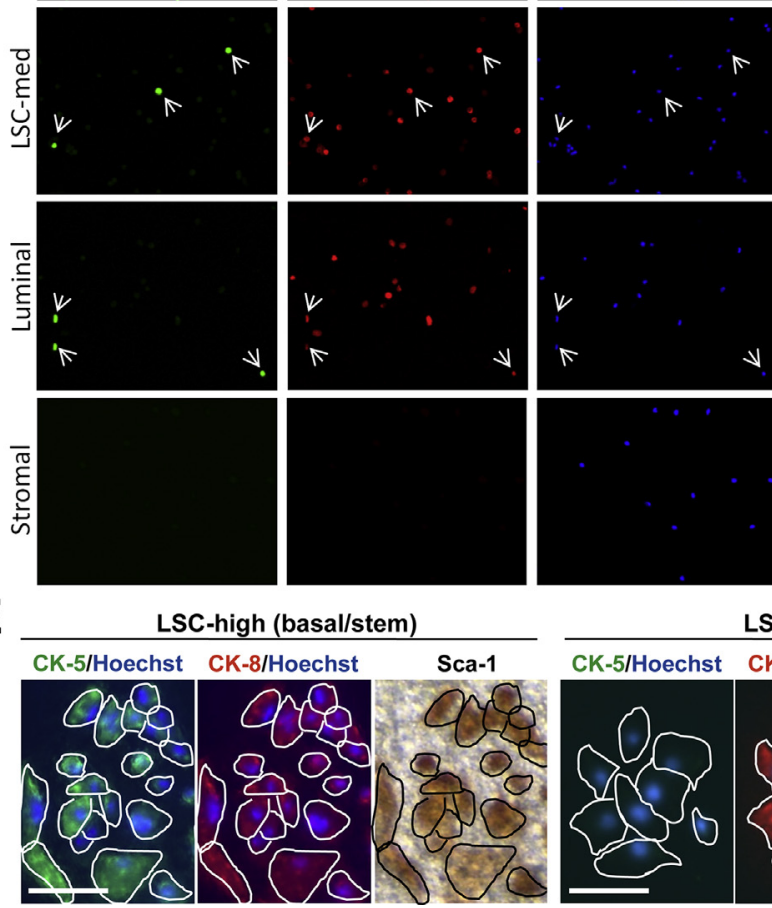
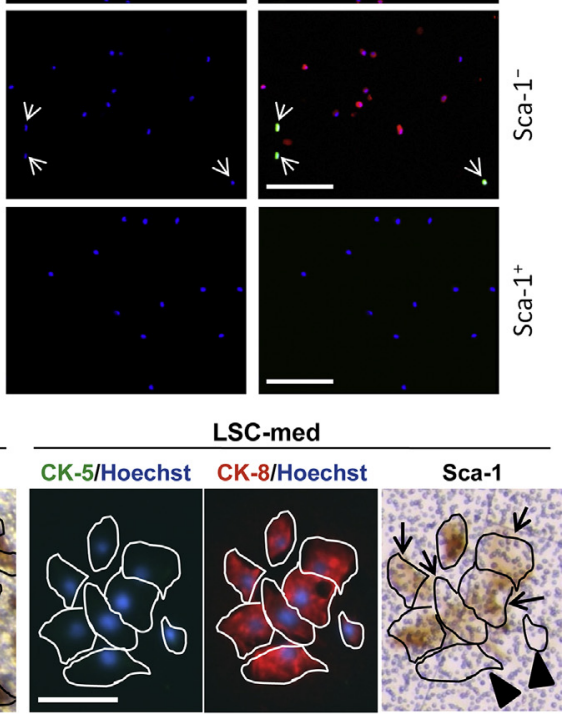

Figure 7 Characterization of sorted subpopulations from $P b-P R L$ mice. A: FACS profile displaying boxed and circled gates used for sorting of each subpopulation. B and C: Sphere generation assay results for sorted LSC-high and LSC-med Pb-PRL subpopulations. B: Spheres generated per 1000 cells seeded for each cell subpopulation. C: Diameter distributions for spheres generated from LSC-high and LSC-med sorted cells. Sphere cross-sectional areas were calculated by ImageJ and converted to diameter values. For reliable comparisons, only experiments producing $>35$ spheres were included. D: Representative images of CK-5 and CK- 8 immunofluorescent costaining performed on cytospin slides of sorted $P b-P R L$ prostate subpopulations. Double positive $\mathrm{CK}-5^{+} \mathrm{CK}-8^{+}$cells are marked with arrows. Labels on the right indicate which sorted subpopulations were positive or negative for Sca-1. Similar results were obtained in three independent experiments. E: Representative images of epithelial colonies from sorted LSC-high and LSCmed cells grown on Matrigel-coated wells and costained for CK-5 and CK-8 by IF and Sca- 1 by immunocytochemistry. White and black outlines delimit individual cells. Arrowheads indicate Sca-1- ${ }^{-}$cells; arrows indicate cells displaying intermediate Sca-1 expression. Similar results were obtained in two independent experiments. $n=6$ independent experiments (B); $n=4$ independent experiments for LSC-high spheres (C); $n=2$ for LSC-med spheres $(\mathbf{C}) .{ }^{*} P<0.05$ by paired $t$-test (B); $P<0.001$ by a $\chi^{2}$ test for trend between distributions (C). Scale bars: $100 \mu \mathrm{m}$ (D and E). Original magnification: $\times 10$ (D and $\mathbf{E})$. other subpopulations. Depending on sorting yields, between 10,000 and 60,000 cells from each subpopulation were loaded in three-dimensional culture plates at a density of 5000 to 10,000 cells/well. Even though overall sphere yields varied from one experiment to another, LSC-high (basal/ stem) cells always gave origin to significantly more spheres than LSC-med cells (Figure 7B). This was consistent with the literature ${ }^{8,20}$ indicating that the LSC-high subpopulation was highly enriched in stem cells. Interestingly, LSC-med cells gave rise to large spheres $(>120 \mu \mathrm{m})$ more frequently than LSC-high cells (Figure 7C), suggesting that LSC-med cells might contain highly proliferating progenitor cells or progenitor cells that are more responsive to the growth factors present in the culture medium. To complement these findings, we returned to the series of basal cell cluster areas analyzed by IF/IHC on tissue slides and determined the presence of $\mathrm{Ki}-67$ staining in basal versus luminal epithelial layers within these areas. For luminal cells we discriminated between Sca- $1^{-}$(mature luminal cells) and Sca- ${ }^{+}$staining (putative LSC-med cells). Positive Ki-67 staining was detected more frequently in Sca- $1^{+}$luminal layers $(52 \%)$, followed by basal cell layers (36\%), and finally by $\mathrm{Sca}^{-}{ }^{-}$luminal layers (7\%) (Supplemental Figure S4). Figure $5 \mathrm{H}$ shows a representative $\mathrm{Sca}-1^{+}$ luminal layer $\left(\mathrm{CK}-5^{-} \mathrm{CK}-8^{+}\right)$displaying positive $\mathrm{Ki}-67$ staining. Together our results indicated that, in addition to amplified basal/stem cells, $P b-P R L$ mouse prostates may be enriched in a putative subpopulation of luminal progenitors that express Sca-1 and might display enhanced proliferation.

Finally, to test whether LSC-med cells were capable of differentiating into $\mathrm{Sca}-1^{-}$luminal cells in vitro, we plated these cells in a colony assay under $5 \alpha$-dihydrotestosterone stimulation. Under these conditions, LSC-med colonies displayed a mixture of Sca- $1^{+}$and Sca- $1^{-}$cells, with some 
cells showing intermediate levels of Sca-1 (Figure 7E). These results suggested that LSC-med cells can differentiate into Sca- $1^{-}$luminal cells in vitro. LSC-high colonies, in contrast, were mainly composed of $\mathrm{CK}-5^{+}$or double CK$5^{+} \mathrm{CK}-8^{+}$cells with high or intermediate levels of Sca-1 (Figure 7E).

\section{Discussion}

The PRL/JAK2/STAT5 pathway is emerging as a putative target for alternative treatment of prostate cancer. ${ }^{1,9,10}$ The present study aimed to characterize the reported amplification of mouse prostate basal/stem cells induced by local PRL, ${ }^{3}$ given that these cells have been proposed to be the initiators of prostate cancer and to promote tumor cell survival and disease recurrence after androgen withdrawal. ${ }^{7}$ Our results indicated that the development of basal/stem cell clusters in $P b-P R L$ mouse prostates was associated with the activation of PRL/Stat5 signaling. More importantly, PRL also led to the amplification of luminal Sca- $1^{+}$cells, which could represent putative progenitor cells giving rise to tumors in the prostate epithelium.

Our previous report ${ }^{3}$ indicated that basal/stem cells were amplified in $P b-P R L$ mice as observed by IHC staining with p63. We now confirmed these findings with the use of FACS, indicating that sorted LSC-high cells (usually called LSC cells), which are enriched for basal/stem epithelial cells, ${ }^{20}$ were amplified in $P b-P R L$ prostates. Basal cell amplification with appearance of basal cell clusters have also been reported for prostate-specific PTEN-deficient $\left(P T E N^{--}\right)$ mice, ${ }^{15}$ which model the loss of the tumor suppressor PTEN, highly prevalent in human prostate cancer. ${ }^{21}$ In $P b-P R L$ mice, a global analysis of ventral, lateral, and dorsal prostate lobes indicated that basal cell clusters as well as Stat5 activation were more prevalent in dorsal lobes than in ventral lobes. The prevalence of hyperplastic foci (including PIN lesions) followed the same trend, whereas weight gain and epithelial proliferation rates were similar across lobes. These results suggest that disorganized hyperplasia and the development of basal cell clusters in $P b-P R L$ mice are directly linked to Stat 5 activation rather than to a general increase in cell proliferation. This idea is supported by the finding that epithelial areas surrounding basal cell clusters in $P b-P R L$ prostates frequently displayed high nuclear Stat 5 staining (Figure 5A). Interestingly, given that Stat5 activation was not found in basal/stem cells but only in adjacent luminal cells, the amplification of basal/stem cells via PRL/Stat5 signaling likely involves paracrine mechanisms that remain to be elucidated.

The lack of detection of any activated PRLR signaling pathways in ventral lobes as opposed to dorsolateral lobes in $P b-P R L$ mice is intriguing. Previous data indicated differences in the response to PRL in rodent prostate lobes, suggesting a more marked effect of PRL in dorsal and lateral lobes than in the ventral lobe. ${ }^{2,12,13}$ However, our data indicate that acute PRL stimulation activates the same signaling pathway (Jak2/Stat5) in all three prostate lobes. Therefore, the observed phenotypic differences among these lobes in $P b-P R L$ mice likely reflect distinct degrees of sustained activation of this signaling cascade rather than activation of alternative PRLR-stimulated pathways. The mechanisms leading to the decreased PRL sensitivity in the ventral prostate of $P b-P R L$ mice probably involve the decreased expression of the long PRLR isoform in this lobe. The underlying cause of this decrease remains to be established. Available data from rat prostate organ cultures suggest a positive regulation of PRLR levels by PRL in rat dorsal and lateral lobes (unfortunately, ventral lobes were not included in that study). ${ }^{22}$ However, given the high level of PRL-transgene expression detected in the ventral lobe of $P b-P R L$ mice, only a negative regulation would be consistent with the decrease in ventral PRLR expression. To confirm and further study the mechanisms underlying the decreased response to PRL in the ventral lobe, time course analyses could be performed on $P b-P R L$ mice at young ages (when transgene expression begins) and/or WT mice under prolonged PRL stimulation. We would expect a decreased response to gradually develop in the ventral lobe with advancing age or time of stimulation for $P b-P R L$ and WT mice, respectively. These studies should help establish key players involved in the down-regulation of the response to PRL, which may have therapeutic interest for current efforts to target the PRLR/STAT5 pathway in human prostate cancer. Overall, the marked activation of PRL/Stat5 signaling and higher prevalence of tissue alterations observed in the $P b-P R L$ dorsal lobe suggest that, among mouse prostate lobes, the dorsal lobe is more appropriate to model human prostate cancer, particularly the $>50 \%$ of human prostate tumors that display PRL expression and activation of STAT5.

In addition to Stat5 activation, high Sca-1 staining and presence of intermediate $\mathrm{CK}-5^{+} \mathrm{CK}-8^{+}$cells were more prevalent in basal cell cluster areas of $P b-P R L$ mice than of WT mice, suggesting variations in differentiation status among basal cell cluster areas of each genotype. The presence of intermediate cells in the $P b-P R L$ model might reflect the differentiation of stem/progenitor $\mathrm{CK}-5^{+}$cells into cells of the luminal lineage. Interestingly, cells coexpressing basal and luminal CKs are also amplified in $P T E N^{-/-}$ prostates $^{23}$ and are present in human prostate cancer. ${ }^{24}$ In fact, we had previously reported an increase in the expression of the stem/progenitor cell marker Sca-1 in $P b-P R L$ dorsal prostates, ${ }^{3}$ but the identity of the epithelial cells expressing this marker had not been analyzed. We now show that not only basal-like cells but also luminal-like cells express Sca-1 in the $P b-P R L$ prostate epithelium, as evidenced by FACS and IF/IHC.

Our results found close associations among high Sca-1 staining, high proliferation, and presence of intermediate cells within basal cell cluster areas of $P b-P R L$ prostate tissue. However, colocalization of these markers on individual 


\begin{tabular}{ccccc}
\multicolumn{2}{c}{ Basal compartment $-\mathrm{CK}-5^{+}$} & Luminal compartment $-\mathrm{CK}-8^{+}$ \\
\hline \multirow{2}{*}{ Stem cell } & Transit-amplifying cell & $\begin{array}{c}\text { Intermediate } \\
\text { cell }\end{array}$ & $\begin{array}{c}\text { Luminal } \\
\text { progenitor }\end{array}$ & Luminal cell
\end{tabular}

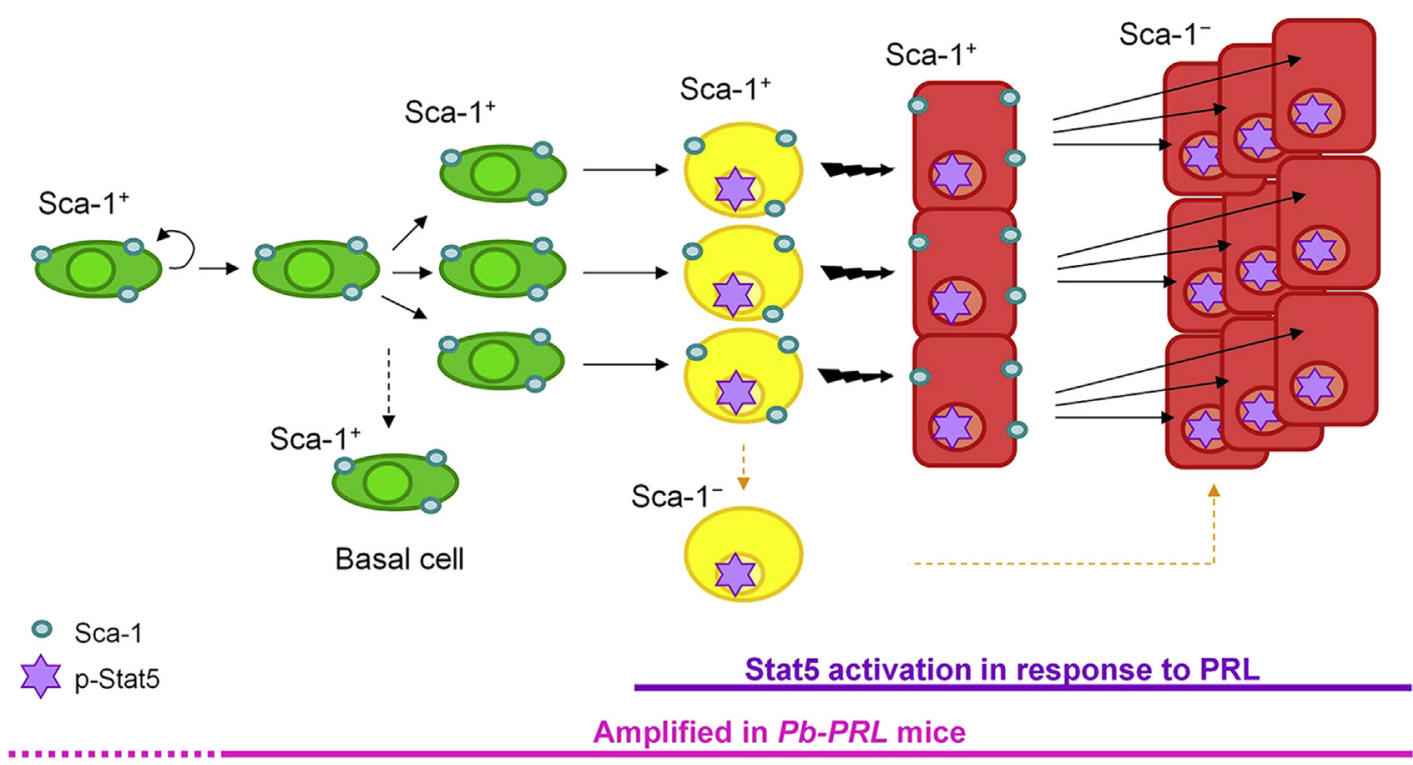

Figure 8 Proposed model of prostate epithelial hierarchy according to our results from Pb-PRL mice. PRL induces the amplification of basal, intermediate, and luminal cells, including a subpopulation of Sca- $1^{+}$luminal cells that we propose to be progenitors. The amplification of the basal compartment involves basal progenitors and might include stem cells as well (dashed pink line). Amplified intermediate cells originated from basal progenitors rapidly differentiate into luminal Sca- $1^{+}$progenitors (lightning-shaped arrows symbolize the rapidity of this process). In turn, these luminal progenitors give rise to mature luminal cells. Our results from sorted prostate subpopulations also indicated the existence of Sca- $1^{-}$intermediate cells. We propose that these cells originate from Sca- $1^{+}$intermediate cells and that they subsequently differentiate into luminal cells (orange dashed arrows). All intermediate and luminal cells can respond to PRL stimulation with $\mathrm{p}$-Stat5 and translocation into the nucleus (purple stars). Finally, the existence of terminally differentiated basal cells originating from basal progenitors was neither established nor denied by our results (black dashed arrow). CK- $5^{+}$cells are green; CK- $8^{+}$cells are red; double CK $-5^{+}$CK- $-8^{+}$intermediate cells are yellow. The membrane marker Sca-1 is represented as blue circles on the membrane of Sca- $1^{+}$cells.

cells cannot be assured from our analysis. The fact that intermediate cells were detected among sorted Sca- $1^{+}$ (LSC-high and LSC-med) and Sca- $1^{-}$(luminal) subpopulations (Figure 7D) suggests that some but not all of these cells might express the Sca-1 marker. Moreover, given the overall low frequency of detection of intermediate cells in basal cell cluster areas, we hypothesize that these intermediate cells probably differentiate rapidly and therefore represent a brief state in the prostate epithelial cell lineage. In addition, differentiation of intermediate cells into Sca- $1^{+}$ luminal-like-amplifying cells would explain the association found between the presence of intermediate cells and higher proliferation within the luminal layers of the basal cell cluster areas analyzed. A model illustrating these considerations is presented in Figure 8.

We have found an amplified subpopulation of $\mathrm{Lin}^{-} \mathrm{Sca}-$ $1^{+} \mathrm{CD} 49 \mathrm{f}^{\text {med }}$ (LSC-med) cells in $P b-P R L$ mouse prostates which had not been previously described for WT mice. Analysis of CK expression and sphere-generating capacity suggest that LSC-med cells represent a subpopulation of putative luminal $\left(\mathrm{CK}-8^{+}\right)$progenitors (only progenitors and stem cells being capable of generating spheres ${ }^{25}$ ). Preliminary experiments indicated lower self-renewal capacity for LSC-med spheres, although they were similar to LSChigh spheres in terms of CKs and p63 expression/localization (data not shown). Interestingly, spheres generated from LSC-med cells were frequently larger than spheres generated from LSC-high cells. This nicely correlated with the frequently positive $\mathrm{Ki}-67$ staining of Sca- ${ }^{+}$luminal cells observed in our IHC analysis of basal cell cluster areas. Finally, in vitro tests confirmed that LSC-med cells were able to differentiate into $\mathrm{Sca}-1^{-}$luminal cells. Thus, it seems reasonable to conclude that LSC-med cells represent highly proliferating $\mathrm{Sca}_{-}{ }^{+}$luminal progenitor cells. Figure 8 shows a model of prostate epithelial hierarchy that is based on our results in $P b-P R L$ mice. Several epithelial cell lineage hierarchy models have been proposed for the adult prostate. ${ }^{26-28}$ Our observations are in good agreement with the model presented by Isaacs, ${ }^{28}$ except that we have introduced a rapidly proliferating luminal progenitor population as discussed. Lineage tracing studies will be necessary to establish whether these LSC-med cells originate from the differentiation of amplified LSC-high (basal/stem) cells in vivo. The amplification and subsequent differentiation of basal/stem/progenitor cells in $P b-P R L$ prostates could be at the origin of tumor development in this model, 
especially given the reported role of basal/stem cells as cells of origin for prostate cancer (which is normally of luminal phenotype).

The discussed similarities of our $P b-P R L$ model and the prostate-specific $P T E N^{-/-}$model raise interesting questions as to whether some of the mechanisms of tumorigenesis might be common in these two models. One important difference between these models is the rate of tumor progression, which is markedly faster in $P T E N^{-1-}$ prostates, with PIN development at 6 weeks and invasive adenocarcinomas at 9 weeks of age ${ }^{23}$ compared with PIN development at 6 months and occasional adenocarcinomas at 20 months of age in the Pb-PRL model. ${ }^{3}$ At the molecular level, there is no evidence that the similar but substantially slower tumor generation process induced by local PRL shares common mechanisms with those resulting from $P T E N$ deletion. For instance, we found no clear activation of the phosphatidylinositol 3-kinase/Akt pathway in $P b-P R L$ mice compared with WT mice. Reciprocally, we are not aware of reports that support activation of the Stat5 pathway in $P T E N^{-/-}$prostate tumors. The tumor-promoting role of Stat5 has been widely found in various models of established prostate cancer. For example, expression of a dominant negative Stat5b mutant in cell lines derived from the transgenic adenocarcinoma of mouse prostate model decreased cell growth in soft agar and tumor formation in nude mice. ${ }^{29}$ Similarly, forced expression of STAT5 in the CWR22Rv human prostate cancer cell line promoted clonogenic cell survival in vitro and growth of xenografted cells in vivo, whereas forced expression of a dominant negative STAT5 isoform had opposite effects. ${ }^{30}$ However, transgenic mice overexpressing WT Stat5 or constitutively active forms of Stat5 are lacking to support the intrinsic oncogenic properties of this pathway in the mouse prostate. On the basis of our study, we speculate that the contribution of PRLR/Stat5 signaling to prostate tumorigenesis in vivo might be primarily through the up-regulation of stem/progenitor cell populations, that is, the host cells for oncogenic hits that are necessary for initiating prostate cancer. ${ }^{7}$

\section{Conclusions}

Our data indicated that the tumorigenic effects of PRL, including hyperplastic foci and basal cell clusters, were associated with activated Stat5 signaling rather than to a general increase in proliferation in prostate lobes of $P b-P R L$ mice. Detailed analysis of basal cell cluster areas confirmed the link between basal/stem cell amplification and Stat5 activation and suggested the participation of yet unknown paracrine mechanisms that are currently under investigation in our group. Increased progenitor markers and proliferation rates were also observed in basal cell cluster areas of $P b-P R L$ prostates compared with WT prostates. Finally, an amplified subpopulation of putative luminal progenitors, which might play an important role in the process of prostate tumorigenesis induced by PRL was detected in $P b-P R L$ prostates. These results provide further understanding of the action of PRL in the prostate to help establish the utility of this hormone or its downstream signaling pathways as putative alternative targets for prostate cancer treatment.

\section{Acknowledgments}

We thank the Histology Facility, Cellular Imaging Facility, and Animal Facility at the Structure Fédérative de Recherche (SFR) Necker that contributed to this study. We thank Virginie Goubert and Aline Paradis (PerkinElmer) for their help concerning the use and optimization of AlphaScreen technology, Vincent Rouet and Elodie Rambaud for punctual experimental contributions to this study, and JacquesEmmanuel Guidotti for critical review of the manuscript.

\section{Supplemental Data}

Supplemental material for this article can be found at http://dx.doi.org/10.1016/j.ajpath.2014.07.020.

\section{References}

1. Goffin V, Hoang DT, Bogorad RL, Nevalainen MT: Prolactin regulation of the prostate gland: a female player in a male game. Nat Rev Urol 2011, 8:597-607

2. Ahonen TJ, Harkonen PL, Rui H, Nevalainen MT: PRL signal transduction in the epithelial compartment of rat prostate maintained as long-term organ cultures in vitro. Endocrinology 2002, 143:228-238

3. Rouet V, Bogorad RL, Kayser C, Kessal K, Genestie C, Bardier A, Grattan DR, Kelder B, Kopchick JJ, Kelly PA, Goffin V: Local prolactin is a target to prevent expansion of basal/stem cells in prostate tumors. Proc Natl Acad Sci U S A 2010, 107:15199-15204

4. Li H, Ahonen TJ, Alanen K, Xie J, LeBaron MJ, Pretlow TG, Ealley EL, Zhang Y, Nurmi M, Singh B, Martikainen PM, Nevalainen MT: Activation of signal transducer and activator of transcription 5 in human prostate cancer is associated with high histological grade. Cancer Res 2004, 64:4774-4782

5. Li H, Zhang Y, Glass A, Zellweger T, Gehan E, Bubendorf L, Gelmann EP, Nevalainen MT: Activation of signal transducer and activator of transcription-5 in prostate cancer predicts early recurrence. Clin Cancer Res 2005, 11:5863-5868

6. Dagvadorj A, Collins S, Jomain JB, Abdulghani J, Karras J, Zellweger T, Li H, Nurmi M, Alanen K, Mirtti T, Visakorpi T, Bubendorf L, Goffin V, Nevalainen MT: Autocrine prolactin promotes prostate cancer cell growth via Janus kinase-2-signal transducer and activator of transcription-5a/b signaling pathway. Endocrinology 2007, 148:3089-3101

7. Goldstein AS, Huang J, Guo C, Garraway IP, Witte ON: Identification of a cell of origin for human prostate cancer. Science 2010, 329: $568-571$

8. Lawson DA, Zong Y, Memarzadeh S, Xin L, Huang J, Witte ON: Basal epithelial stem cells are efficient targets for prostate cancer initiation. Proc Natl Acad Sci U S A 2010, 107:2610-2615

9. Jacobson EM, Hugo ER, Borcherding DC, Ben-Jonathan N: Prolactin in breast and prostate cancer: molecular and genetic perspectives. Discov Med 2011, 11:315-324

10. Damiano JS, Wasserman E: Molecular pathways: blockade of the PRLR signaling pathway as a novel antihormonal approach for the treatment of breast and prostate cancer. Clin Cancer Res 2013, 19: $1644-1650$ 
11. Ittmann M, Huang J, Radaelli E, Martin P, Signoretti S, Sullivan R, Simons BW, Ward JM, Robinson BD, Chu GC, Loda M, Thomas G, Borowsky A, Cardiff RD: Animal models of human prostate cancer: the consensus report of the New York meeting of the Mouse Models of Human Cancers Consortium Prostate Pathology Committee. Cancer Res 2013, 73:2718-2736

12. Robertson FG, Harris J, Naylor MJ, Oakes SR, Kindblom J, Dillner K, Wennbo H, Tornell J, Kelly PA, Green J, Ormandy CJ: Prostate development and carcinogenesis in prolactin receptor knockout mice. Endocrinology 2003, 144:3196-3205

13. Ahonen TJ, Harkonen PL, Laine J, Rui H, Martikainen PM, Nevalainen MT: Prolactin is a survival factor for androgen-deprived rat dorsal and lateral prostate epithelium in organ culture. Endocrinology 1999, 140:5412-5421

14. Kindblom J, Dillner K, Sahlin L, Robertson F, Ormandy C, Tornell J, Wennbo H: Prostate hyperplasia in a transgenic mouse with prostate-specific expression of prolactin. Endocrinology 2003, 144: $2269-2278$

15. Corbacho AM, Valacchi G, Kubala L, Olano-Martin E, Schock BC, Kenny TP, Cross CE: Tissue-specific gene expression of prolactin receptor in the acute-phase response induced by lipopolysaccharides. Am J Physiol Endocrinol Metab 2004, 287:E750-E757

16. Lukacs RU, Goldstein AS, Lawson DA, Cheng D, Witte ON: Isolation, cultivation and characterization of adult murine prostate stem cells. Nat Protoc 2010, 5:702-713

17. Ben-Jonathan N, LaPensee CR, LaPensee EW: What can we learn from rodents about prolactin in humans? Endocr Rev 2008, 29:1-41

18. Perrot-Applanat M, Gualillo O, Pezet A, Vincent V, Edery M, Kelly PA: Dominant negative and cooperative effects of mutant forms of prolactin receptor. Mol Endocrinol 1997, 11:1020-1032

19. Xin L, Lawson DA, Witte ON: The Sca-1 cell surface marker enriches for a prostate-regenerating cell subpopulation that can initiate prostate tumorigenesis. Proc Natl Acad Sci U S A 2005, 102:6942-6947
20. Lawson DA, Xin L, Lukacs RU, Cheng D, Witte ON: Isolation and functional characterization of murine prostate stem cells. Proc Natl Acad Sci U S A 2007, 104:181-186

21. Phin S, Moore MW, Cotter PD: Genomic rearrangements of PTEN in prostate cancer. Front Oncol 2013, 3:240

22. Nevalainen MT, Valve EM, Ingleton PM, Harkonen PL: Expression and hormone regulation of prolactin receptors in rat dorsal and lateral prostate. Endocrinology 1996, 137:3078-3088

23. Wang S, Garcia AJ, Wu M, Lawson DA, Witte ON, Wu H: Pten deletion leads to the expansion of a prostatic stem/progenitor cell subpopulation and tumor initiation. Proc Natl Acad Sci U S A 2006, 103:1480-1485

24. Schalken JA, van Leenders G: Cellular and molecular biology of the prostate: stem cell biology. Urology 2003, 62(5 suppl 1):11-20

25. Pastrana E, Silva-Vargas V, Doetsch F: Eyes wide open: a critical review of sphere-formation as an assay for stem cells. Cell Stem Cell 2011, 8:486-498

26. Goldstein AS, Stoyanova T, Witte ON: Primitive origins of prostate cancer: in vivo evidence for prostate-regenerating cells and prostate cancer-initiating cells. Mol Oncol 2010, 4:385-396

27. Shen MM, Abate-Shen C: Molecular genetics of prostate cancer: new prospects for old challenges. Genes Dev 2010, 24:1967-2000

28. Isaacs JT: Prostate stem cells and benign prostatic hyperplasia. Prostate 2008, 68:1025-1034

29. Kazansky AV, Spencer DM, Greenberg NM: Activation of signal transducer and activator of transcription 5 is required for progression of autochthonous prostate cancer: evidence from the transgenic adenocarcinoma of the mouse prostate system. Cancer Res 2003, 63: 8757-8762

30. Dagvadorj A, Kirken RA, Leiby B, Karras J, Nevalainen MT: Transcription factor signal transducer and activator of transcription 5 promotes growth of human prostate cancer cells in vivo. Clin Cancer Res 2008, 14:1317-1324 